authorB.I. Ermolaev

\title{
Synthesis of DGLAP and total resummation of leading logarithms for the non-singlet spin structure function $g_{1}$
}

\author{
M. Greco \\ Department of Physics and INFN, University Rome III, Rome, Italy \\ S.I. Troyan \\ St.Petersburg Institute of Nuclear Physics, 188300 Gatchina, Russia
}

\begin{abstract}
The explicit expressions for the non-singlet DIS structure function $g_{1}$ at small $x$ are obtained by resumming the leading logarithmic contributions. The role played by the fits for the initial parton densities currently used in the DGLAP on the small- $x$ behavior of the non-singlet $g_{1}$ is discussed. Explicit expressions combining DGLAP with our results are presented.

PACS numbers: $12.38 . \mathrm{Cy}$
\end{abstract}

\section{INTRODUCTION}

The non-singlet component of the spin structure function $g_{1}$ have been investigated in great detail in deep inelastic scattering (DIS) experiments. The standard theoretical framework for studying the DIS structure functions is provided by DGLAP [1]. In this approach, $g_{1}^{N S}\left(x, Q^{2}\right)$ can be represented as a convolution of the coefficient functions and the evolved quark distributions. Combining these results with appropriate fits for the initial quark distributions, provides a good agreement with the available experimental data.

However, the DGLAP evolution eqs. were originally applied in a range of large $x$ values, where higher-loop contributions to the coefficient functions and the anomalous dimensions are small. Such corrections are becoming essential when $x$ is decreasing, so DGLAP should not work so well at $x \ll 1$. Nevertheless, DGLAP predictions are in a good agreement with available experimental data. It leads to the conclusion that the impact of the higher-order corrections is negligibly small for the available values of $x$. Below we use our results [2] to show that the impact of the high-order corrections on the $Q^{2}$ and $x$-evolutions of the non-singlet structure functions is quite sizable and bounds the region of strict applicability of DGLAP to $x>10^{-2}$. We also show that the reason for the success of DGLAP at $x<10^{-2}$ is related to the sharp $x$-dependence assumed for the initial parton densities, which is able to mimic the role of high-order corrections.

The paper is organized as follows: In Sect. 2 we discuss the difference of our approach with DGLAP. Then we compare our and the DGLAP formulae for asymptotics of $g_{1}$. In Sect. 3 we suggest a method to combine DGLAP with our approach in order to obtain equally correct expressions for both large and small values of $x$. Sect. 4 contains our conclusions.

\section{COMPARISON OF DGLAP AND OUR APPROACH}

As the DGLAP -expressions for the non-singlet structure functions are well-known, we discuss them briefly only. In this approach, $g_{1}^{N S}{ }_{D L A P}\left(x, Q^{2}\right)$ can be represented as a convolution

$$
g_{1 D G L A P}^{N S}\left(x, Q^{2}\right)=\int_{x}^{1} \frac{d y}{y} C(x / y) \Delta q\left(y, Q^{2}\right)
$$

of the coefficient functions $C(x)$ and the evolved quark distributions $\Delta q\left(x, Q^{2}\right)$. Similarly, $\Delta q\left(x, Q^{2}\right)$ can be expressed through the convolution of the splitting functions and the initial quark densities $\delta q\left(x \approx 1, Q^{2} \approx \mu^{2}\right)$ where $\mu^{2}$ is the starting point of the $Q^{2}$-evolution. It is convenient to represent $f\left(x, Q^{2}\right)$ in the integral form, using the Mellin transform:

$$
g_{1 D G L A P}^{N S}\left(x, Q^{2}\right)=\left(e_{q}^{2} / 2\right) \int_{-\imath \infty}^{\imath \infty} \frac{d \omega}{2 \imath \pi}(1 / x)^{\omega} C(\omega) \delta q(\omega) \exp \left[\gamma(\omega) \int_{\mu^{2}}^{Q^{2}} \frac{d k_{\perp}^{2}}{k_{\perp}^{2}} \alpha_{s}\left(k_{\perp}^{2}\right)\right]
$$


where $C(\omega)$ are the non-singlet coefficient functions, $\gamma(\omega)$ the non-singlet anomalous dimensions and $\delta q(\omega)$ the Mellin transforms of the initial non-singlet quark densities. The standard DGLAP fits $\delta q(x)$ for the non-singlet parton densities (see e.g. Refs. 3, 4]) consist of the terms singular when $x \rightarrow 0$ and the regular in $x$ part. For example, the fit A of Ref. [3] is chosen as follows:

$$
\begin{aligned}
& \delta q(x)=N \eta x^{-\alpha} \phi(x), \\
& \phi(x) \equiv(1-x)^{\beta}\left(1+\gamma x^{\delta}\right),
\end{aligned}
$$

with $N, \eta$ being the normalization, $\alpha=0.576, \beta=2.67, \gamma=34.36$ and $\delta=0.75$. As the term $x^{-\alpha}$ in the rhs of Eq. (3) is singular when $x \rightarrow 0$ whereas the second one, $\phi(x)$ is regular, we will address them as the singular and regular parts of the fit respectively. Obviously, in the $\omega$-space Eq. (3) is a sum of the pole contributions:

$$
\delta q(\omega)=N \eta\left[(\omega-\alpha)^{-1}+\sum_{k=1}^{\infty} m_{k}\left((\omega+k-\alpha)^{-1}+\gamma(\omega+k+1-\alpha)^{-1}\right)\right]
$$

with $m_{k}=\beta(\beta-1) . .(\beta-k+1) / k$ !, so that the first term in Eq. (4) (the leading pole) corresponds to the singular term $x^{-\alpha}$ of Eq. (3) and the second term, i.e. the sum of the poles, corresponds to the interference between the singular and regular terms. In contrast to the leading pole position $\omega=\alpha$, all other poles in Eq. (4) have negative values because $k-\alpha>0$. An alternative approach was used in Refs. [? ], by introducing and solving infrared evolution equations with fixed $\alpha_{s}$. This approach was improved in Refs. [2], where single-logarithmic contributions were also accounted for and the QCD coupling was regarded as running in all Feynman graphs contributing to the non-singlet structure functions. In contrast to the DGLAP parametrization $\alpha_{s}=\alpha_{s}\left(k_{\perp}^{2}\right)$, we used in Refs. [2] another parametrization where the argument of $\alpha_{s}$ in the quark ladders is given by the time-like virtualities of the intermediate gluons. Refs. [2] suggest the following formulae for the non-singlet structure functions:

$$
g_{1}^{N S}\left(x, Q^{2}\right)=\left(e_{q}^{2} / 2\right) \int_{-\imath \infty}^{\imath \infty} \frac{d \omega}{2 \pi \imath}(1 / x)^{\omega} C_{N S}(\omega) \delta q(\omega) \exp \left(H_{N S}(\omega) y\right),
$$

with $y=\ln \left(Q^{2} / \mu^{2}\right)$ so that $\mu^{2}$ is the starting point of the $Q^{2}$-evolution. The new coefficient function $C_{N S}$ are expressed in terms of new anomalous dimensions $H_{N S}$ whereas $H_{N S}$ account for the total resummation of the doubleand single- logarithmic contributions (see Ref. [2] for details).

\section{COMPARISON OF DGLAP AND OUR SMALL- $x$ ASYMPTOTICS}

When $x \rightarrow 0$, one can use the saddle point method in order to estimate the integrals in Eq. (5) and derive much simpler expressions for the non-singlet structure functions:

$$
g_{1}^{N S} \sim e_{q}^{2} \delta q\left(\omega_{0}\right) \xi^{\omega_{0}}
$$

with $\xi=\sqrt{Q^{2} /\left(x^{2} \mu^{2}\right)}$ and with the intercept $\omega_{0}=0.42$. Eq. (6) predicts the asymptotic scaling for the non-singlet structure functions: Asymptotically, $g_{1}^{N S}$ depends on one argument $\xi$ instead of depending on $x$ and $Q^{2}$ separately.

When the standard DGLAP fits, e.g. the fit of Eq. (3), are used, the asymptotics of $g_{1}^{N S}{ }_{D L A P}\left(x, Q^{2}\right)$ is also the Regge-like:

$$
g_{1 D G L A P}^{N S} \sim\left(e_{q}^{2} / 2\right) C(\alpha)(1 / x)^{\alpha}\left(\left(\ln \left(Q^{2} / \Lambda^{2}\right)\right) /\left(\ln \left(\mu^{2} / \Lambda^{2}\right)\right)\right)^{\gamma(\alpha) / b}
$$

with $b=\left(33-2 n_{f}\right) / 12 \pi$.

Comparison of Eq. (6) and Eq. (7) demonstrates that both DGLAP and our approach lead to the Regge asymptotic behavior in $x$. However, it is important that our intercept $\omega_{0}$ is obtained by the total resummation of the leading logarithmic contributions and without any assumption about fits for $\delta q$ whereas the DGLAP intercept $\alpha$ in Eq. (7) is generated by the phenomenological factor $x^{-0.57}$ of Eq. (3) which mimics the total resummation. In other words, the impact of the higher-loop radiative corrections on the small- $x$ behavior of the non-singlets is, actually, incorporated into DGLAP phenomenologically, through the fits. It means that the singular factors can be dropped from such fits when the coefficient function includes the total resummation of the leading logarithms and therefore in this case fits for $\delta q$ can be chosen as regular functions of $x$. 


\section{COMBINING DGLAP WITH OUR HIGHER-LOOP CONTRIBUTIONS}

Eq. (5) accounts for the resummation of the double- and single logarithmic contributions to the non-singlet anomalous dimensions and the coefficient functions that are leading when $x$ is small. However, the method we have used does not allow us to account for other contributions which can be neglected for $x$ small but become quite important when $x$ is not far from 1 . On the other hand, such contributions are naturally included in DGLAP, where the non-singlet coefficient function $C_{D G L A P}$ and anomalous dimension $\gamma_{D G L A P}$ are known with the two-loop accuracy:

$$
\begin{aligned}
& C_{D G L A P}=1+\frac{\alpha_{s}\left(Q^{2}\right)}{2 \pi} C^{(1)} \\
& \gamma_{D G L A P}=\frac{\alpha_{s}\left(Q^{2}\right)}{4 \pi} \gamma^{(0)}+\left(\frac{\alpha_{s}\left(Q^{2}\right)}{4 \pi}\right)^{2} \gamma^{(1)}
\end{aligned}
$$

Therefore, we can borrow from the DGLAP formulae the contributions which are missing in Eq. (5) by adding $C_{D G L A P}$ and $\gamma_{D G L A P}$ to the coefficient function and anomalous dimension of Eq. (5). It is important to avoid a double counting DL and SL terms common for these expressions.

In order to do so, let us consider the region of $x \sim 1$ where the effective values of $\omega$ in Eqs. (25) are large. In this region we can expand $H_{N S}$ and $C_{N S}$ into a series in $1 / \omega$. Retaining the first two terms in each series, we arrive at $C_{N S}=\widetilde{C}_{N S}+O\left(\alpha_{s}^{2}\right), H_{N S}=\widetilde{H}_{N S}+O\left(\alpha_{s}^{3}\right)$, with (see Ref. [2] for details)

$$
\begin{aligned}
\widetilde{C}_{N S} & =1+\frac{A(\omega) C_{F}}{2 \pi}\left[1 / \omega^{2}+1 / 2 \omega\right], \\
\widetilde{H}_{N S} & =\frac{A(\omega) C_{F}}{4 \pi}[2 / \omega+1]+\left(\frac{A(\omega) C_{F}}{4 \pi}\right)^{2}(1 / \omega)[2 / \omega+1]^{2}+D[1 / \omega+1 / 2] .
\end{aligned}
$$

Now let us define the new coefficient functions $\hat{C}_{N S}$ and new anomalous dimensions $\hat{C}_{N S}$ as follows:

$$
\begin{aligned}
& \hat{H}_{N S}=\left[H_{N S}-\widetilde{H}_{N S}\right]+\frac{A(\omega)}{4 \pi} \gamma^{(0)}+\left(\frac{A(\omega)}{4 \pi}\right)^{2} \gamma^{(1)}, \\
& \hat{C}_{N S}=\left[C_{N S}^{( \pm)}-\widetilde{C}_{N S}\right]+1+\frac{A(\omega)}{2 \pi} C^{(1)} .
\end{aligned}
$$

These new, "synthetic" coefficient functions and anomalous dimensions of Eq. (10) include both the total resummation of the leading contributions and the DGLAP expressions in which $\alpha_{s}\left(Q^{2}\right)$ is replaced by $A(\omega)$ defined in Refs. [2] because the factorization of the phase space into transverse and longitudinal spaces used in DGLAP to parametrize $a l p h a_{s}$ is a good approximation for large $x$ only.

[1] G. Altarelli and G. Parisi, Nucl. Phys., B126 (1977) 297; V.N. Gribov and L.N. Lipatov, Sov. J. Nucl. Phys. textbf15 (1972) 438; L.N.Lipatov, Sov. J. Nucl. Phys. 20 (1972) 95; Yu.L. Dokshitzer, Sov. Phys. JETP 46 (1977) 641.

[2] B.I. Ermolaev, M. Greco and S.I. Troyan, Nucl.Phys. B594B (2001)71; ibid B571(2000)137; Phys.Lett. B579, 321,(2004); hep-ph/0503019

[3] G. Altarelli, R.D. Ball, S. Forte and G. Ridolfi, Nucl. Phys. B496 (1997) 337; Acta Phys. Polon. B29(1998)1145;

[4] A. Vogt. hep-ph/0408244 